\title{
Cefditoren Pivoxil
}

National Cancer Institute

\section{Source}

National Cancer Institute. Cefditoren Pivoxil. NCI Thesaurus. Code C47434.

A semi-synthetic, broad-spectrum, beta-lactamase resistant, third-generation cephalosporin antibiotic with bactericidal activity. Cefditoren pivoxil is a prodrug that is rapidly hydrolyzed by intestinal esterases during absorption to the microbiologically active cefditoren, an active aminothiazolyl cephalosporin. Cefditoren inactivates penicillin binding proteins (PBPs) thereby interfering with peptidoglycan synthesis and inhibiting bacterial cell wall synthesis. Another consequence of beta-lactam exposure results in the loss of lipoteichoic acids from the cell wall. Lipoteichoic acids inhibit murein hydrolase activity and their absence from the cell wall triggers uncontrolled autolytic activity rendering bacterial cells susceptible to osmotic shock. This results in a reduction of cell wall stability and causes cell lysis. 